\title{
Probing the Electron Beam Induced Structural Evolution of Halide Perovskite Thin Films by Scanning Transmission Electron Microscopy
}

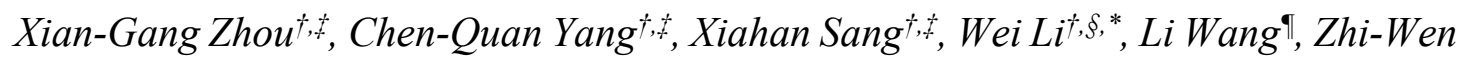

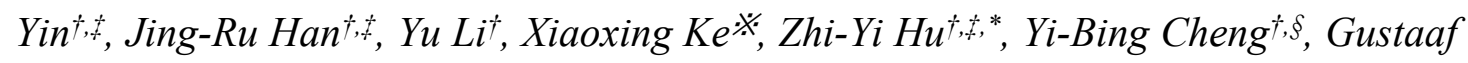
Van Tendeloo ${ }^{\dagger, t, * * *}$

† State Key Laboratory of Advanced Technology for Materials Synthesis and Processing, Wuhan University of Technology, Luoshi Road 122, Wuhan 430070, China

NRC (Nanostructure Research Centre), Wuhan University of Technology, Luoshi Road 122, Wuhan 430070, China

$\S$ Foshan Xianhu Laboratory of the Advanced Energy Science and Technology Guangdong Laboratory, Xianhu hydrogen Valley, Foshan 528200, China

『 Materia Nova R\&D Center, Avenue du champ de Mars 6, Mons 7000, Belgium

※ Beijing Key Laboratory of Microstructure and Properties of Solids, Faculty of Materials and Manufacturing, Beijing University of Technology, Pingleyuan 100, Beijing 100124, China

** EMAT (Electron Microscopy for Materials Science), University of Antwerp, Groenenborgerlaan 171, Antwerp 2020, Belgium

*Corresponding author (wei.li@whut.edu.cn, zhiyi.hu@whut.edu.cn) 


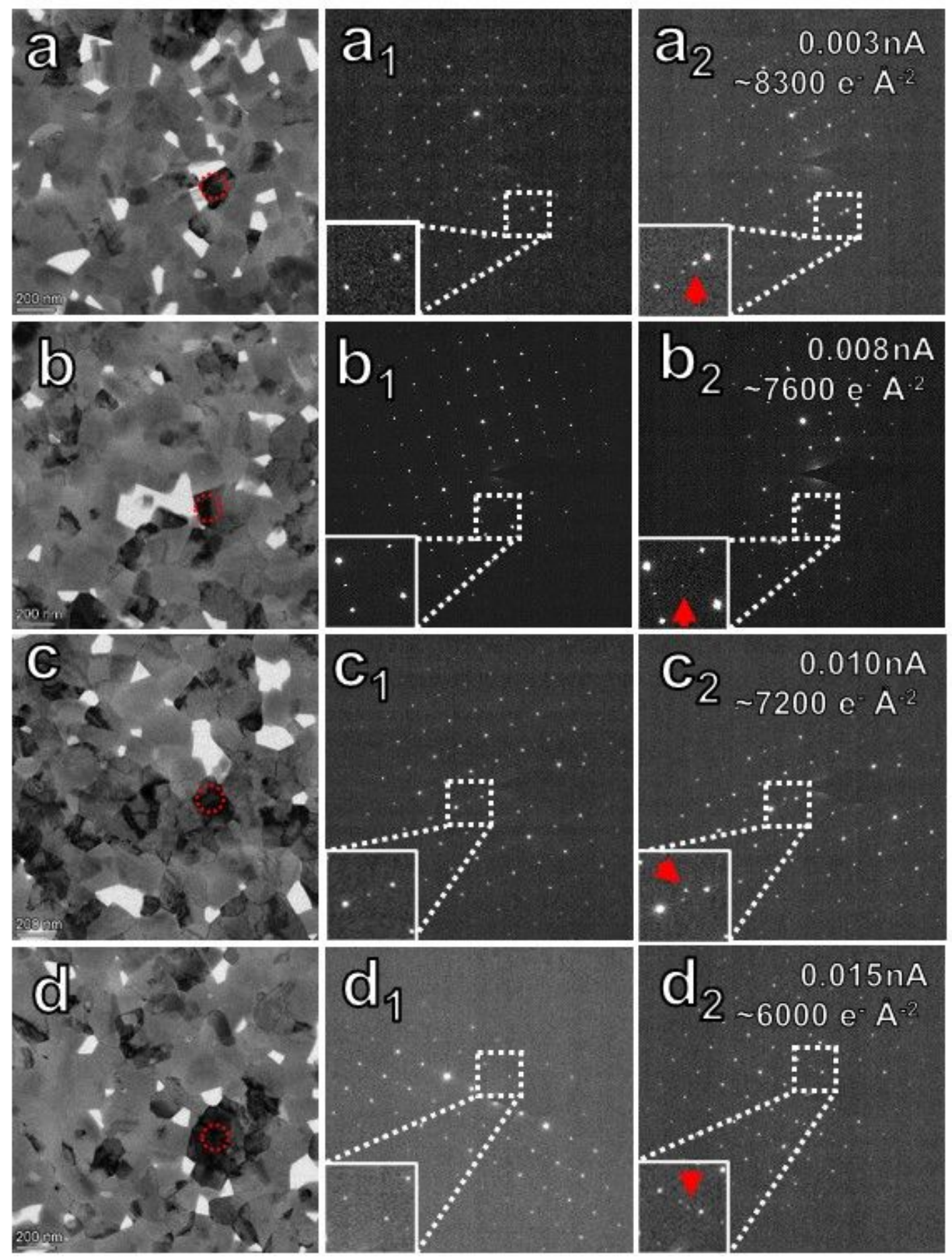

Figure.S1 (a-d) Low magnification TEM images, $\left(a_{1}-d_{1}\right)$ corresponding SAED patterns of the pristine $\gamma-C s \mathrm{PbIBr}_{2}$ grains indicated by red dashed circles in $(\mathrm{a}-\mathrm{h}),\left(\mathrm{a}_{2}-\mathrm{d}_{2}\right)$ the first observation of the forbidden reflections in the SAED patterns with the dose increasing (indicated by red arrows). 


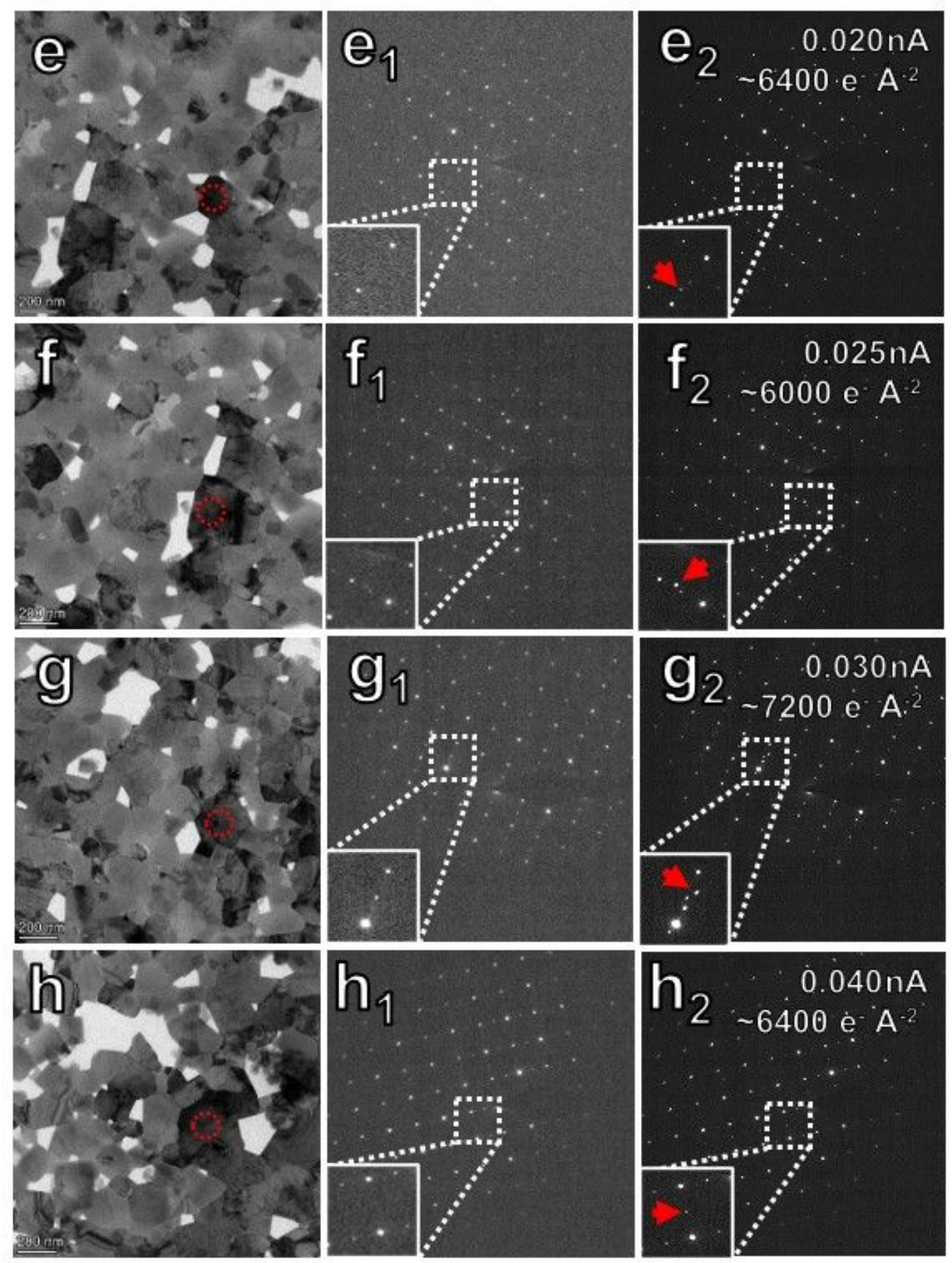

Figure.S2 (e-h) Low magnification TEM images, $\left(\mathrm{e}_{1}-\mathrm{h}_{1}\right)$ corresponding SAED patterns of the pristine $\gamma-\mathrm{Cs} \mathrm{PbIBr}_{2}$ grains indicated by red dashed circles in (e-h), ( $\mathrm{e}_{2}-$ $\mathrm{h}_{2}$ ) the first observation of the forbidden reflections in the SAED patterns with the dose increasing (indicated by red arrows). 

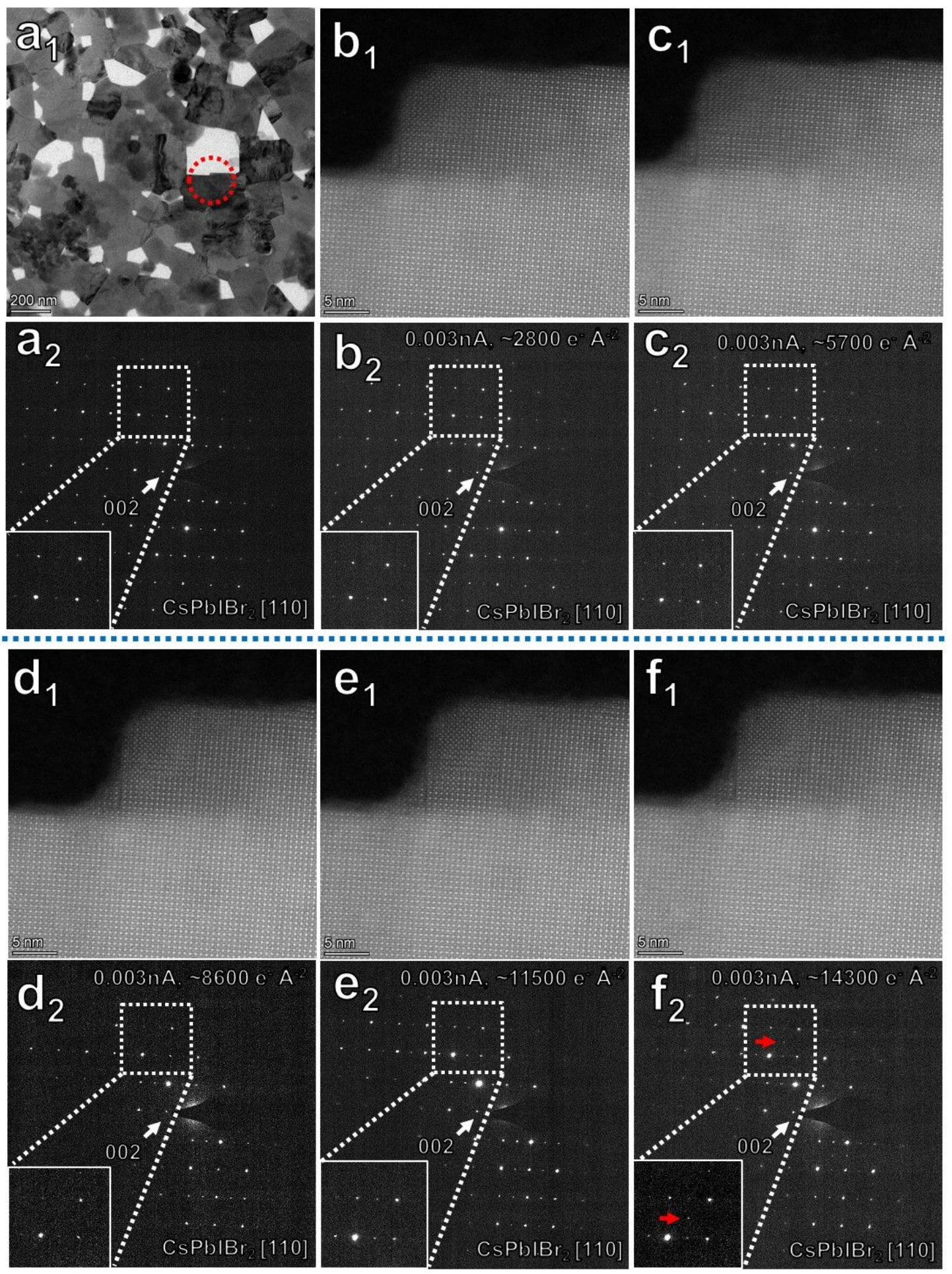

Figure.S3 $\left(a_{1}\right)$ Low magnification TEM image of the original $\gamma-\mathrm{CsPbIBr}_{2}$ thin film, $\left(\mathrm{a}_{2}\right)$ corresponding SAED pattern of the grain indicated by red dashed circles, $\left(b_{1}-f_{1}\right)$ HRHAADF-STEM images with the accumulative overall-dose increasing intermittently before every 5 minutes pause in Figure. $2 \mathrm{f},\left(\mathrm{b}_{2}-\mathrm{f}_{2}\right)$ corresponding SAED patterns showing additional diffraction dots indicated by red arrows. 


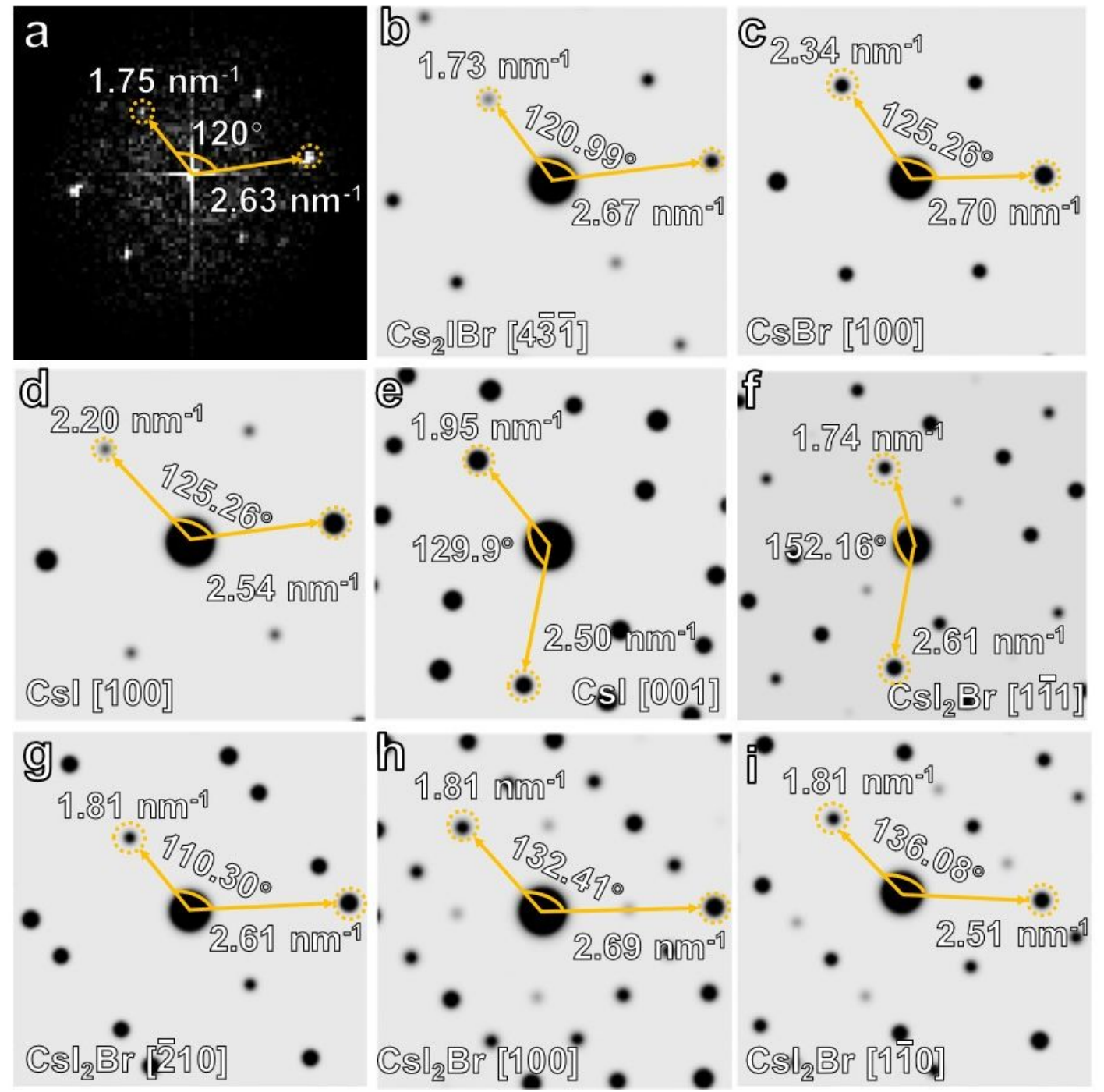

Figure.S4 (a) FFT pattern of Figure.4 c (inset), (b) simulated diffraction pattern of $\mathrm{Cs}_{2} \mathrm{IBr}$ along the [4-3-1] zone axis, (c-i) simulated diffraction patterns of other crystal phases ( $\mathrm{Cs}_{2} \mathrm{IBr}, \mathrm{CsBr}, \mathrm{CsI}$ and $\left.\mathrm{CsI}_{2} \mathrm{Br}\right)$ along possible zone axes; corresponding angles and distances of crystal planes are indicated. 
Table S1 Recommended experimental parameters for inorganic halide perovskite materials in STEM mode

\begin{tabular}{|c|c|c|c|c|c|c|}
\hline $\begin{array}{c}\text { Probe } \\
\text { curren } \\
\mathbf{t} \\
(\mathbf{n A}) \\
\end{array}$ & $\begin{array}{c}\text { Dwell } \\
\text { time } \\
(\mu s)\end{array}$ & $\begin{array}{c}\text { Frame } \\
\text { time } \\
(\mathrm{s})\end{array}$ & $\begin{array}{c}\text { Frame } \\
\text { resolution }\end{array}$ & $\begin{array}{l}\text { Pixel } \\
\text { size } \\
(\AA)\end{array}$ & $\begin{array}{c}\text { Image } \\
\text { size } \\
(\AA)\end{array}$ & $\begin{array}{c}\text { Overall dose } \\
\text { per image } \\
\left(\mathrm{e}^{-/} \AA^{2}\right)\end{array}$ \\
\hline \multicolumn{7}{|c|}{ Suggested experimental parameters with safe working condition in STEM } \\
\hline 0.0001 & 10 & 11.7 & $1024 \times 1024$ & 0.1711 & 175.21 & 238.2133 \\
\hline 0.003 & 1 & 1.57 & $1024 \times 1024$ & 0.2419 & 247.71 & 479.7658 \\
\hline 0.008 & 10 & 11.7 & $512 \times 512$ & 2.712 & 1388.5 & 303.4147 \\
\hline 0.010 & 5 & 23.5 & $2048 \times 2048$ & 0.2419 & 495.41 & 5984.37 \\
\hline 0.015 & 10 & 45.2 & $2048 \times 2048$ & 2.712 & 5554.2 & 137.3632 \\
\hline \multicolumn{7}{|c|}{ No suggested experimental parameters resulting in beam damage in STEM } \\
\hline 0.008 & 10 & 11.7 & $1024 \times 1024$ & 0.2419 & 247.71 & 9534.199 \\
\hline 0.010 & 5 & 23.5 & $2048 \times 2048$ & 0.1711 & 350.41 & 11961.56 \\
\hline 0.015 & 10 & 45.2 & $1024 \times 1024$ & 0.2419 & 247.71 & 69059.37 \\
\hline 0.020 & 5 & 23.5 & $2048 \times 2048$ & 0.1711 & 350.41 & 23923.13 \\
\hline 0.025 & 10 & 11.7 & $1024 \times 1024$ & 0.1711 & 175.21 & 59550.87 \\
\hline 0.030 & 10 & 11.7 & $1024 \times 1024$ & 0.2419 & 247.71 & 35753.25 \\
\hline 0.040 & 10 & 11.7 & $1024 \times 1024$ & 0.2419 & 247.71 & 479671 \\
\hline
\end{tabular}




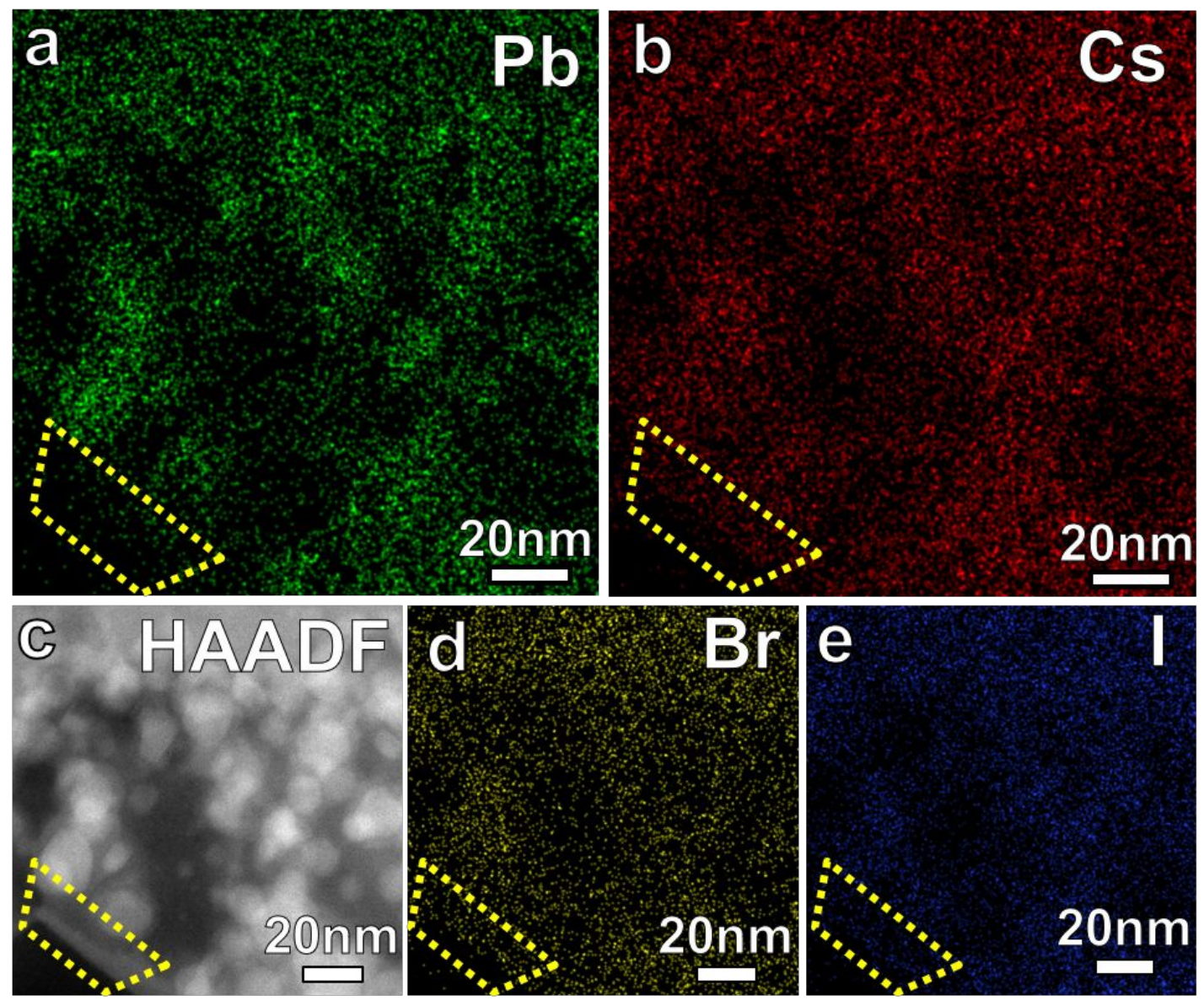

Figure.S5 (a-e) Magnified HAADF-STEM and corresponding EDS elemental maps in Figure.4, and the corresponding edge area of Figure.4c indicated by the yellow dashed box.

Table S2 The EDS elemental quantification of the area indicated by the yellow dashed box in Figure.S4

\begin{tabular}{|c|c|c|c|}
\hline $\mathrm{Z}$ & Element & Family & Atomic fraction (\%) \\
\hline 35 & $\mathrm{Br}$ & $\mathrm{K}$ & 52.63 \\
\hline 53 & $\mathrm{I}$ & $\mathrm{L}$ & 14.57 \\
\hline 55 & $\mathrm{Cs}$ & $\mathrm{L}$ & 24.74 \\
\hline 82 & $\mathrm{~Pb}$ & $\mathrm{~L}$ & 3.06 \\
\hline
\end{tabular}




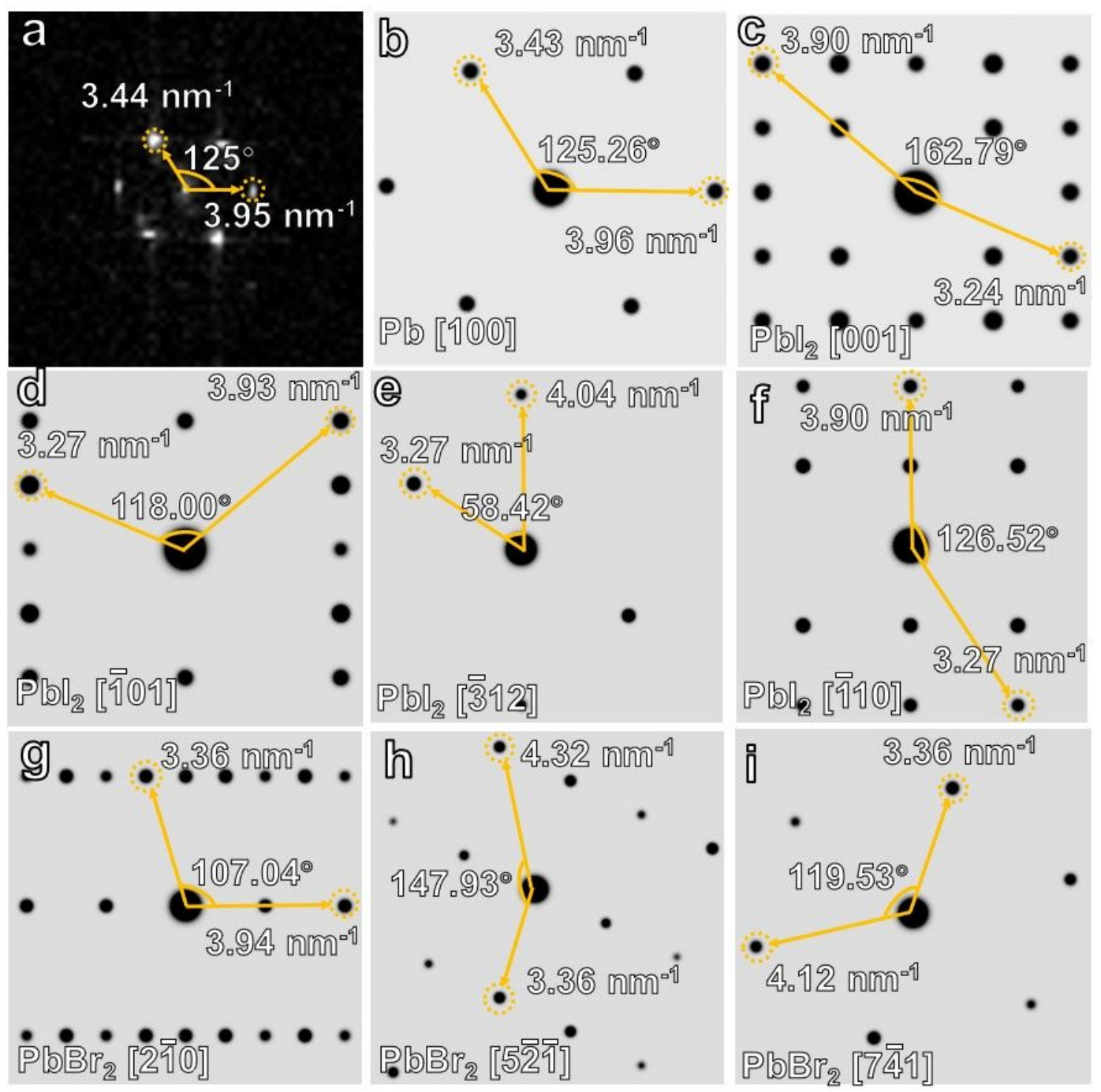

Figure.S6 (a) FFT pattern of Figure.4 b inset, (b) simulated diffraction pattern of $\mathrm{Pb}$ along the [100] zone axis, (c-i) simulated diffraction patterns of crystal phases $\left(\mathrm{PbI}_{2}\right.$ and $\mathrm{PbBr}_{2}$ ) along possible zone axes; the corresponding angles and distances of crystal planes are indicated. 
Table S3 The reference overall dose of different main (S)TEM techniques with different modes

\begin{tabular}{|c|c|c|}
\hline Technique & Mode & Overall Dose $\left(\mathrm{e}^{-} / \AA^{2}\right)$ \\
\hline \multirow{2}{*}{ Diffraction } & Normal imaging ${ }^{1}$ & $10^{1}-10^{4}$ \\
\hline & Low dose imaging ${ }^{1,2} 3$ & $10^{-1}-10^{1}$ \\
\hline \multirow{3}{*}{ TEM } & Normal imaging 2,4 & $10^{3}-10^{4}$ \\
\hline & Low dose imaging ${ }^{2} 5,6$ & $1 \times 10^{2}-8 \times 10^{3}$ \\
\hline & Direct electronic camera imaging 7 & $1-10$ \\
\hline \multirow{3}{*}{ STEM } & Normal imaging ${ }^{8}$ & $10^{4}-2 \times 10^{5}$ \\
\hline & Low dose imaging ${ }^{8}$ & $2 \times 10^{2}-2 \times 10^{4}$ \\
\hline & iDPC imaging $5,9,10$ & $10^{1}-10^{3}$ \\
\hline \multirow{2}{*}{ EDS } & Spectrum ${ }^{11}$ & $10^{3}-10^{5}$ \\
\hline & Mapping $^{11}$ & $\geq 9 \times 10^{4}$ \\
\hline \multirow{2}{*}{ EELS } & Spectrum $^{12}$ & $1.5 \times 10^{3}-3.6 \times 10^{8}$ \\
\hline & Mapping ${ }^{12}$ & $\geq 4 \times 10^{5}$ \\
\hline
\end{tabular}




\section{References:}

(1). Kodjikian, S.;Klein, H., Low-dose electron diffraction tomography (LD-EDT). Ultramicroscopy 2019, 200, 12-19.

(2). Rothmann, M. U.;Li, W.;Zhu, Y.;Liu, A.;Ku, Z.;Bach, U.;Etheridge, J.;Cheng, Y. B., Structural and Chemical Changes to $\mathrm{CH}_{3} \mathrm{NH}_{3} \mathrm{PbI}_{3}$ Induced by Electron and Gallium Ion Beams. Adv Mater 2018, 30 (25), e1800629.

(3). Chen, Q.;Dwyer, C.;Sheng, G.;Zhu, C.;Li, X.;Zheng, C.;Zhu, Y., Imaging Beam-Sensitive Materials by Electron Microscopy. Adv Mater 2020, 32 (16), e1907619.

(4). Williams, D. B.;Carter, C. B., High-Resolution TEM. In Transmission Electron Microscopy: A Textbook for Materials Science, Williams, D. B.; Carter, C. B., Eds. Springer US: Boston, MA, 2009; pp 483-509.

(5). Zhang, D. L.;Zhu, Y. H.;Liu, L. M.;Ying, X. R.;Hsiung, C. E.;Sougrat, R.;Li, K.;Han, Y., Atomicresolution transmission electron microscopy of electron beam-sensitive crystalline materials. Science 2018, 359 (6376), 675-+.

(6). Yu, Y.;Zhang, D. D.;Kisielowski, C.;Dou, L. T.;Kornienko, N.;Bekenstein, Y.;Wong, A. B.;Alivisatos, A. P.;Yang, P. D., Atomic Resolution Imaging of Halide Perovskites. Nano Lett. 2016, 16 (12), 7530-7535.

(7). Zhu, Y.;Ciston, J.;Zheng, B.;Miao, X.;Czarnik, C.;Pan, Y.;Sougrat, R.;Lai, Z.;Hsiung, C. E.;Yao, $\mathrm{K}$., et al., Unravelling surface and interfacial structures of a metal-organic framework by transmission electron microscopy. Nat Mater 2017, 16 (5), 532-536.

(8). Rothmann, M. U.;Kim, J. S.;Borchert, J.;Lohmann, K. B.;O'Leary, C. M.;Sheader, A. A.;Clark, L.;Snaith, H. J.;Johnston, M. B.;Nellist, P. D., et al., Atomic-scale microstructure of metal halide perovskite. Science 2020, 370 (6516).

(9). Liu, L.;Wang, N.;Zhu, C.;Liu, X.;Zhu, Y.;Guo, P.;Alfilfil, L.;Dong, X.;Zhang, D.;Han, Y., Direct Imaging of Atomically Dispersed Molybdenum that Enables Location of Aluminum in the Framework of Zeolite ZSM-5. Angew Chem Int Ed Engl 2020, 59 (2), 819-825.

(10). Shen, B.;Chen, X.;Cai, D.;Xiong, H.;Liu, X.;Meng, C.;Han, Y.;Wei, F., Atomic Spatial and Temporal Imaging of Local Structures and Light Elements inside Zeolite Frameworks. Adv Mater 2020, 32 (4), e1906103.

(11). Song, K.;Liu, L.;Zhang, D.;Hautzinger, M. P.;Jin, S.;Han, Y., Atomic-Resolution Imaging of Halide Perovskites Using Electron Microscopy. Adv. Energy Mater. 2020, 10 (26).

(12). Jiang, N.;Spence, J. C. H., On the dose-rate threshold of beam damage in TEM. Ultramicroscopy 2012, 113, 77-82. 